\title{
Structural Design Analysis of Vehicle Muffler Based on CFD
}

\author{
Min Yang ${ }^{1, a, *}$, Xiao Zhang ${ }^{2}$ \\ ${ }^{1}$ College of Mechanical Engineering, Nanjing University of Science and Technology ZiJin College, \\ WenlanRoad 89, Qixia District, Nanjing, Jiangsu Province, 210046, China \\ ${ }^{2}$ Nanjing Xingqiao Y-tec Automobile Parts Co. Ltd. No.29 Jingyou Road, Jiangning Science Park, Jiangning \\ District, Nanjing, Jiangsu Province, 210058, China \\ a yangmin082@njust.edu.cn \\ *corresponding author
}

Keywords: CFD, Vehicle muffler, Structural design

\begin{abstract}
With the continuous development of social economy, the speed of automobile replacement is getting faster and faster, not only to improve the speed and safety performance of automobile, but also to pay more attention to the silencing disposal of automobile, to reduce the exhaust noise, to use CFD method to analyze the flow of automobile exhaust gas, to discuss the design optimization scheme of muffler, and then to provide reliable data research for the installation of muffler, and to provide favorable data support for the installation of muffler in the future.
\end{abstract}

\section{Introduction}

With the development of economy, motor vehicles have become the main tool for people's daily life. However, the rapid development of industry has brought difficulties to the development of cities. Some data show that automobile noise has become one of the main sources of urban noise pollution.

\section{Modelling Overview}

There is a lot of noise from the automobile exhaust pipe, including fundamental frequency noise, gas column noise, injection noise Germany, so the setting of automobile silencing can be achieved by means of CFD (Computational Fluid Dynamics) method, quoting the numerical contents of computer and discretization, numerical simulation and analysis of the contents of fluid mechanics, and analyzing the noise of automobile through the obtained numerical contents. Second, ensure that the design has no special impact on engine power. Thus, the sound volume of the sound wave is reduced by the way of reflection and interference in the pipe. In general, the performance of automobile muffler should include three aspects, one acoustic performance, two airflow regeneration noise, and three aerodynamic performance, so as to complete the complex structure involving many disciplines.

\section{The Role of CFD in Muffler}

The influence of CFD on the muffler is roughly divided into the following two aspects, one is the relationship between the internal structure of the muffler and the middle of the airflow, and the temperature and density around it are changed by the propagation medium, and the state of the sound wave in the silencing pipe is different. Second, since the state of the airflow itself will absorb the motion of the surrounding gas or solid to produce new noise, especially when the gas flow velocity is at $10 \mathrm{~m} / \mathrm{s}$, the effect of the airflow on the silencing amount will be minimized, and the resulting silencing amount should be paid more attention to when the gas flow velocity is higher than $10 \mathrm{~m} / \mathrm{s}$.

The design of muffler should also consider different aspects of data content, especially when the 
current back pressure increases, its internal combustion power will decrease, then the air flow pressure through the muffler entrance and exit will also decrease, resulting in pressure loss, its pressure loss has a great relationship with the internal structure of muffler, and also has a close relationship with the air flow velocity. When the air velocity of the inlet and outlet increases, the speed of the pressure loss will be increased quickly, thus increasing the power loss of the engine. Therefore, in the design of the muffler, the muffler should be given a reasonable air velocity.

To sum up, in the process of CFD vehicle muffler structure design, it is necessary to analyze the content of gas flow field by analyzing the aerodynamic performance index, so as to find out the area where the regenerative noise appears, and then optimize the structural design of the whole muffler, and then determine the concrete scheme by comparing the rationality of the different design of the muffler.[1].

There are many tiny holes in the muffler, which can change the structure and vary in thickness. In order to improve the accuracy, the mesh at the perforation is smaller than that at other places, and the fluid domain and solid domain grid of muffler are divided by tetrahedral mesh. In the case of constant parameters of the flow domain and solid domain of the muffler, gravity and fluid medium are not considered, so the muffler is designed.

One of the small holes should not be too small, one is not easy to manufacture, the other is easy to block, affecting the exhaust capacity of the car. At the same time, it is necessary to ensure that the spacing between the small holes is large enough, which will reduce the injection fluid, otherwise it will be pooled into large airflow injection and increase the noise. Therefore, the total opening area is larger than the exhaust pipe cross section area 30\%, otherwise it will affect the exhaust and reduce the engine power.

\section{CFD Simulation and Analysis of Results}

\subsection{Analysis of Pressure Field}

The concept of pressure loss is put forward in the above contents, and its main loss has important relation with the friction pressure caused by the friction between the inner channel wall of the muffler and the air flow, the non-channel bending in the muffler, and the change of section. In general, the pressure loss will be taken into account first, especially the loss study of the diversion pipe, it is necessary to take into account its energy loss content, in the comparison of the difference value of the flow rate of the front and rear diversion pipe, the larger the difference value, the greater the pressure loss will also increase.

As shown in Fig .1(see Fig .1), the muffler pressure distribution is related to the pressure distribution of each cavity. If the pressure between the first and second cavities increases, then the pressure of the other cavities will decrease accordingly, which is related to the expansion of the large cavity hard gas of the tube diameter. If a large volumetric airflow is needed in the first cavity to offset the exhaust pulse of the engine, the equilibrium spatial pressure distribution of the gas parameters will be more averaged to achieve the goal of reducing the pressure loss, so that the pressure loss is compared from the different volumetric cavity design contents[2].

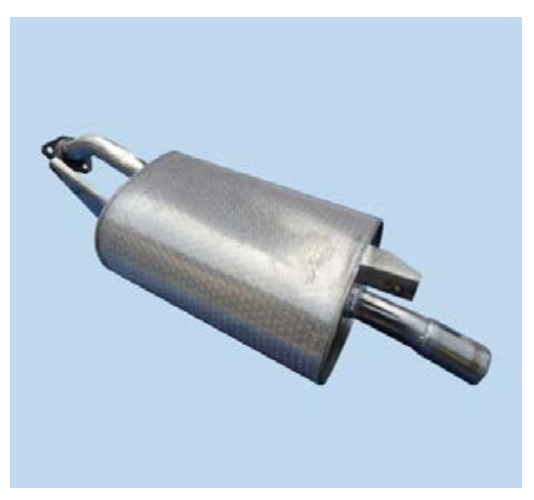

Figure 1 Car muffler 


\subsection{Velocity Flow Field Analysis}

The influence of flow velocity has a great influence on the jet noise, first of all, it will emit turbulent noise because of the rapid injection noise or the different turbulence in the pipe mouth. Since the velocity map is in the same region as the surrounding noise, the influence of the velocity is analyzed in the length of each cavity following its radius. When the air velocity of the local area increases, the surrounding jet noise should be taken into account. If the small scale turbulence near the outlet interacts with the surrounding pipe opening, the turbulent noise will occur. It can be said that when the speed near the outlet of the pipe is large, turbulence will occur around it, resulting in turbulence noise[3]. While the jet noise has a certain radiation characteristic, which is related to its power content, and its sound power is proportional to the airflow velocity 8th power. Therefore, in understanding the content of jet noise, we need to consider the air velocity, if the air velocity is large, then the noise will increase. If the pipe diameter of different diversion pipes is the same as the depth length, it is necessary to compare the turbulence velocity of the different diversion pipes. if the velocity associated between the first cavity and the second cavity is not much different, the eddy current dissipation needs to be reduced in order to reduce the pressure loss, but such a way will not reduce the silencer and produce more kinetic energy, thus increasing the gas jet of the second cavity. if the turbulent design of the orifice plate is carried out for the connected conduit of the third cavity, the velocity of the turbulent dissipation action will be appropriately reduced, and the gas at the outlet will reduce the effect of the jet (see figure ii).

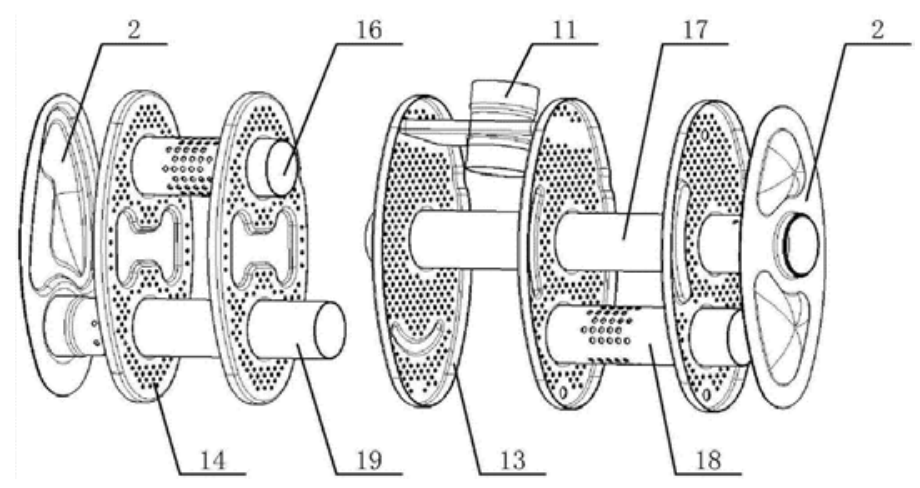

Figure 2 Design of muffler

In fact, eddy current generation is a contradictory body for muffler, eddy current generation is a contradictory body for muffler, only if the eddy current is enhanced, its holy energy can reduce the dissipation, thus reducing the silencer, which will also be the pressure loss increase, resulting in the impact on the engine. Therefore, we should consider the comprehensive design intention, so as to reduce the tendency, use the appropriate eddy current area to reduce the jet and reduce the turbulence noise and injection noise under certain pressure loss.

By analyzing the fluid dynamics of the muffler, it can be found that the hole plate is connected to the cavity through the non-joint body. If the cavity is in the state of streamline disorder, the dissipation of the hole hole is lower, and in the turbulent vortex field, the vortex will produce a series of reactions in the cavity, such as development, formation, shedding and so on, thus forming the turbulent noise. When the vortex falls off, it produces the same pulsating force, the direction of action is consistent with the direction of the flow of air. The sound rate of eddy current noise is proportional to the 6th power of airflow velocity, so, according to this rule, it is necessary to reduce the situation of eddy current at the tail tube, otherwise, it will not be able to reduce the noise condition effectively, and then, the velocity of the tail tube should not exceed $45-50 \mathrm{~m} / \mathrm{s}$.

When the airflow changes the law of sound wave propagation, when the direction of sound wave propagation is opposite to the direction of air flow, the coefficient of muffler will gradually decrease, and the noise reduction will increase[4]. In particular, the tail gas has a certain noise reduction in the muffler folding process, but this folding structure will also moderately increase the exhaust gas emission resistance, so it is necessary to measure the relationship between the two in the process of silencing design (see figure 3). 


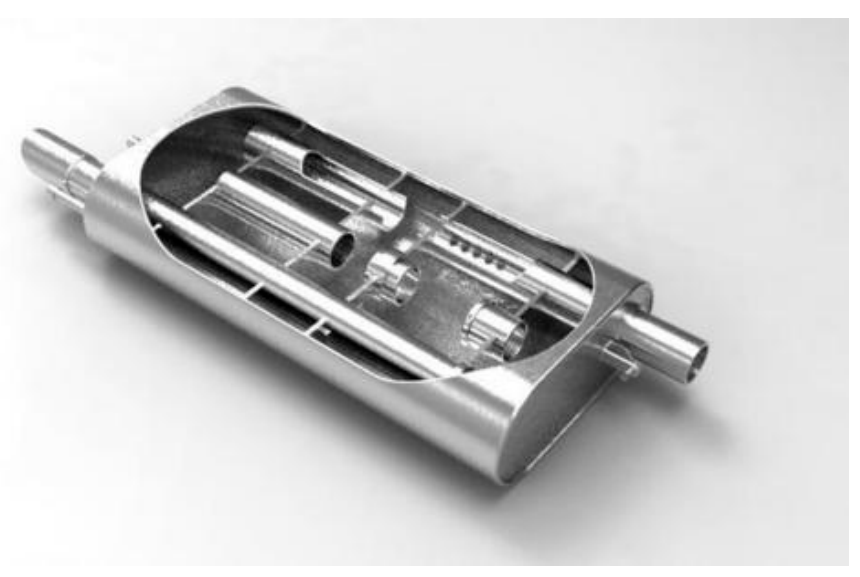

Figure 3 Design of muffler

Exhaust pressure loss is mainly in the high-speed air flow through the interpolation tube, directly hit the wall of the muffler, will be through the way of rebound, so that the muffler into the exhaust pipe. This way will cause serious gas loss, so in the design process, we should increase the crosssectional area when the gas turns, reduce the depth of the muffler in the exhaust pipe, and uniformly reduce the loss of pressure.

Because the air pressure of the intake pipe is large and the air pressure of the exhaust pipe is small, the loss of exhaust pressure will reduce the power of the knife engine, which will increase the perforation rate of the small hole, need to enhance the position and length of the internal intubation, and improve the position of the internal intubation in different ways.

\section{Conclusion}

To sum up, in the muffler angle treatment, the use of circular arc structure for excessive setting, effectively alleviate the resistance of air flow. The use of sound-absorbing materials can enhance the efficacy of mufflers, when the direction of airflow propagation is opposite to the direction of sound wave propagation, the noise reduction will increase, and the exhaust resistance will also increase. Reasonable arrangement of internal intubation and exploring its depth and position can reduce the pressure loss properly without affecting the noise reduction effect of muffler. Through the above contents, we can grasp the muffler's noise attenuation performance, gas power, regeneration noise on the three content, will have different performance and economic effect, using these three aspects of content to develop and debug the muffler by CFD method, but we should pay attention to the calculation method and their respective factors, take CFD as the overall optimization design scheme, which is convenient to calculate the influence of the airflow state of the engine on the muffler in different operation, can effectively reduce the R \& $\mathrm{D}$ cost, shorten the $\mathrm{R} \& \mathrm{D}$ cycle, and get more effective data, thus enhance the actual work demand.

\section{Acknowledgements}

The work described in this paper was supported by the General Natural Science Foundation in 2016 of Jiangsu Educational Committee, PR China, "Research on driving stability and control of tilt steering three wheel ENV" (16KJD460004)

\section{References}

[1] Fu, Yaomin., Luo, Weidong., Zhao, Feihu., Nie, Zhongke. Special vehicle exhaust muffler multi-field study and structural performance analysis. Machine Tools and Hydraulics , vol. 47, no. 19, pp. 114, 2019.

[2] Wang, Chunhua. muffler-triggered automotive fault analysis. Light Vehicle Technology , no. Z3, pp. 71, 2019. 
[3] Tan, Naihu. Development and implementation of high temperature muffler performance test system. Chongqing University, 2019.

[4] Zhang, Zhanwen., Geng, Yanbo., Su, Junhe. Performance analysis and optimization of muffler in engineering vehicles. Construction Machinery , vol. 47, no. 01, pp. 22, 2016. 